\title{
Multihop range-free localization with approximate shortest path in anisotropic wireless sensor networks
}

\author{
Sangwoo Lee ${ }^{1}$, Chansik Park², Min Joon Lee ${ }^{3}$ and Sunwoo Kim ${ }^{1 *}$
}

\begin{abstract}
This paper presents a multihop range-free localization algorithm that tolerates network anisotropy with a small number of anchors. A detoured path detection is proposed to detect if the shortest path between nodes is detoured from their direct path by measuring the deviation in the hop count between the direct and shortest paths. A novel distance estimation method is introduced to approximate the shortest path based on the path deviation and to estimate their distance by taking into account the extent of the detour of the approximate shortest path. Compared to other range-free algorithms, the proposed algorithm requires fewer anchors while achieving higher localization accuracy in anisotropic networks. We demonstrated its superiority over existing range-free localization algorithms through extensive computer simulations.
\end{abstract}

Keywords: Range-free localization; Anisotropic networks; Approximate shortest path; Detoured path detection; Path deviation

\section{Introduction}

In wireless sensor networks (WSNs), localization has received a great deal of attention since it facilitates a variety of applications [1] such as environmental monitoring, health care, target tracking, and military surveillance. This technique is also considered to be a fundamental requirement for broadcasting and routing $[2,3]$ in WSNs. Many localization schemes have been developed to autonomously pinpoint the locations of normal nodes with the assistance of anchors which have perfect location information. These localization schemes fall into rangebased schemes or range-free schemes.

The common feature of range-based localization schemes is that each normal node calculates the distances or directions to the anchors or neighbors based on the following signal measurements [4-8]: received signal strength, time of arrival, time difference of arrival, and/or angle of arrival. The range-based schemes in general provide more accurate location estimates than the range-free schemes; however, the ranging operation leads

*Correspondence: remero@hanyang.ac.kr

1 Department of Electronics and Computer Engineering, Hanyang University, Seoul 133-791, Republic of Korea

Full list of author information is available at the end of the article to an increase in the installation cost and reduction in the network lifetime due to the need of additional hardware of procedure. For these problems that are critical to WSNs with limited resources, the range-based schemes are considered improper solutions to the localization problem in WSNs.

To overcome the drawbacks of the range-based schemes, range-free localization schemes solve the localization problem with network topology information. Conventional range-free approaches $[9,10]$ are focused on finding the most likely area that each node is present. Since these approaches exploit knowledge received from directly heard anchors, it is assumed that a large number of anchors are uniformly deployed or the anchors have transmission radii that are several times larger than those of normal nodes. However, such assumptions lead to cost and scalability problems. In contrast, multihop range-free approaches [11-13] exploit network connectivity information (i.e., the hop count of the shortest path between nodes) to localize normal nodes with a limited number of anchors in large-scale networks. Although these approaches effectively cope with the problems of the one-hop type approaches $[9,10]$, they are applicable only

\section{Springer}

(c) 2014 Lee et al.: licensee Springer. This is an Open Access article distributed under the terms of the Creative Commons Attribution License (http://creativecommons.org/licenses/by/2.0), which permits unrestricted use, distribution, and reproduction in any medium, provided the original work is properly credited. 
to isotropic networks where the hop count of the shortest path between two nodes is assumed to be proportional to the Euclidean distance between them. This assumption implies that all the nodes are uniformly distributed in a regular region and the shortest path between any pair of nodes nearly corresponds to their direct path that forms a straight line connecting the node pair.

Unfortunately, such an assumption no longer holds in anisotropic networks which can be commonly found, and this assumption can be invalidated by a variety of factors: obstacles, resource limitation, sparse and nonuniform node distribution, irregular radio propagation pattern, and irregular regions [14]. These factors make the shortest path between nodes detoured from their direct path, and the hop count measured along the shortest path becomes larger than the expected hop count of the direct path. Moreover, the flip ambiguity problem [15], defined as the phenomenon where the estimated graph of the network is locally translated, rotated, and/or reflected, can arise. To enhance the localization accuracy and to resolve the flip ambiguity problem, recursive refinement algorithms [16,17] were suggested based on the mass spring method which is an optimization tool minimizing the differences between the estimates and local measurements (within few hops). However, the location estimate can oscillate over time and converge to a false minimum unless a sufficient number of anchors are uniformly deployed and consistent link connectivity is guaranteed.

Recently, there have been efforts [14,18-21] to relieve the effects of network anisotropy. Localization using expected hop progress (LAEP) algorithm [18] was suggested to tolerate the anisotropy from nonuniform node distribution. Although the LAEP algorithm provides improved distance estimates for node pairs having slightly detoured paths, its performance significantly deteriorates in irregular-shaped regions where most of the node pairs' shortest paths are heavily detoured. Rendered path [19] was proposed to solve the problem in irregular regions by using a hole detection method [22], which detects nodes at the boundaries of holes. This approach can achieve relatively high localization accuracy with a small number of anchors; however, a large amount of communication overhead is involved in the hole detection, and it is still vulnerable to sparse and nonuniform node deployments and radio irregularity. Reliable anchor selection-based approaches $[14,20,21]$ were proposed to restrict the use of information of anchors that are suspected of having detoured shortest paths. The pattern-driven algorithm [14] uses anchors within 8 hops from a normal node. The reliable anchor-based localization algorithm [20] selects those anchors whose average hop progresses are larger than the minimum hop progresses for the hop counts. The supervised anchor- based algorithm [21] chooses the subset of anchors that minimizes the sum of squared distance estimation errors. These approaches can prevent sources causing considerably large errors from heavily detoured paths. However, the performance of the reliable anchor selection-based algorithms deteriorates dramatically unless a sufficient number of anchors are uniformly deployed. In [23,24], fingerprinting-type approaches were developed, but they also require a number of uniformly deployed anchors for the implementation. On this wise, most of the previous work presumes a large number of anchors to enhance the localization accuracy in anisotropic networks. However, it is costly and inefficient to deploy numerous anchors to solve the localization problem in WSNs because anchors are equipped with global navigation satellite system receivers, which are expensive and relatively high-power-consuming devices, to acquire their locations.

This paper presents a distributed multihop range-free localization algorithm to mitigate the effects of network anisotropy with a small number of anchors. A detoured path detection method is presented to detect whether or not the shortest path between nodes is detoured by measuring the path deviation between the direct and shortest paths of a node pair. A distance estimation is proposed to estimate the distance between nodes with their approximate shortest path based on the path deviation. With the approximate shortest path, the proposed algorithm enables each normal node to measure how much the shortest path to an anchor is detoured from the direct path and to estimate the distance more accurately.

The remainder of the paper is organized as follows. Section 2 describes the network model and terminologies used in this paper. Section 3 introduces a novel multihop range-free localization algorithm. Section 4 presents the experimental results of the proposed algorithm in comparison with other existing algorithms. We conclude the paper in Section 5.

\section{Network model}

Consider a WSN in the two-dimensional space in which any regulations in spacing or pattern of the nodes are undefined. All the nodes including normal nodes and anchors are unable to measure the distances or directions to other nodes, and they are randomly deployed. Once these nodes are deployed, they periodically emit hello packets with identifications to advertise their existence to adjacent nodes. Each anchor broadcasts a beacon message containing its location and hop count set to zero. When a node receives the beacon message, it increments the hop count value in the message by one and the updated message is forwarded to its neighbors. With this process (generally called flooding or broadcasting [2]), all the 
nodes in the network obtain the locations of the anchors and the hop counts measured along the shortest paths to them.

Suppose that a WSN consists of $N$ normal nodes and $A$ anchors $(A \ll N)$. The sets of normal nodes and anchors are, respectively, denoted by $\Omega_{N}$ and $\Omega_{A}$. The nodes are assumed to be homogeneous, stationary, and equipped with omnidirectional antennas with a maximum transmission radius $d_{\max }$. The transmission radius is unstable due to the radio irregularity, also known as the degree of irregularity (DOI). The quasi unit disk graph (QUDG) communication model [25] is assumed, such that two nodes build a perfect link if $d \in\left[0, d_{\max } / \mathrm{DOI}\right]$ or a link with probability $p=\operatorname{DOI}\left(d_{\max }-d\right) / d_{\max }(\mathrm{DOI}-1)$ if $d \in\left(d_{\max } / \mathrm{DOI}, d_{\max }\right]$ where $d$ is the Euclidean distance between the nodes and DOI $>1$. Let $\mathbf{p}_{i}=\left[x_{i}, y_{i}\right]^{T}$ be the $x-y$ coordinates of node $i$. The distance between nodes $i$ and $j$ is $d_{i j}=\left\|\mathbf{p}_{i}-\mathbf{p}_{j}\right\|$, where $\|\cdot\|$ is the 2D Euclidean norm. Let $h_{i j}$ be the hop count measured along the shortest path between nodes $i$ and $j$.

\section{Multihop range-free localization with approximate shortest path}

\subsection{Overview}

This section presents a multihop range-free localization algorithm that estimates the distance between an anchor and a normal node by taking into account how much their shortest path is detoured from their direct path. Before introducing the proposed algorithm, we address the detoured path detection problem.

According to [20], the shortest path from any node to an anchor, having a small average hop progress, is judged to be detoured. However, the judgement may be wrong since the small average hop progress of the anchor only indicates that the anchor has detoured paths to other anchors [11]. In fact, the detoured path detection problem can be readily solved if the expected hop count of the direct path between nodes is given; the shortest path between nodes is considered detoured if the hop count of the shortest path is larger than the expected hop count. Unfortunately, the expected hop count of the direct path between any node pair is unidentifiable. Besides, nodes are unable to estimate their expected hop count due to lack of information on their direct path (e.g., the Euclidean distance). Therefore, the detoured path detection problem for an anchor-to-normal node pair cannot be solved by itself.

Suppose that the expected hop counts between anchors are known (we propose a method to derive the expected hop count between anchors in this paper). Then, the detoured path detection can be performed for an anchor pair's shortest path. Motivated by this, we solve the detoured path detection problem for an anchor-to-normal node pair by transforming into the problem for an anchorto-normal-to-anchor pair. From the viewpoint of a normal node observing two anchors, the shortest path between the anchor pair is regarded as the path connecting the shortest paths from the normal node to the anchors, and their hop count is calculated as the sum of the separately measured hop counts to the anchors. Define the shortest path of an anchor-to-normal-to-anchor pair as the compound shortest path of the anchor pair for clarity. By comparing the hop counts of the direct and compound shortest paths between the anchors, the normal node is able to judge whether the compound shortest path is detoured.

Again, to solve the detoured path detection problem for an anchor-to-normal node pair, we approximate the anchor pair's compound shortest path to bypass a virtual hole between the anchors based on the path deviation (i.e., the difference between the expected hop count and the hop count of the compound shortest path). In this paper, a virtual hole is modeled as a circular-shaped hole. By approximating the compound shortest path along the boundary of the virtual hole, the normal node is now able to detect whether the shortest path to each anchor is detoured and to estimate the distance to the anchor by taking into account the extent of the detour of the path. Therefore, our problem is to generate a virtual hole based on the path deviation of an anchor pair. The idea of the proposed algorithm is illustrated in Figure 1.

\subsection{Detoured path detection for compound shortest path}

Consider that normal node $i$ observes an anchor pair $j$ and $k$. Let $h_{i(j, k)}=h_{i j}+h_{i k}$ be the hop count of the compound shortest path, observed at normal node $i$, between anchors $j$ and $k$. Denote the expected hop count between the anchors by $h_{j k}^{*}$. The normal node can easily detect whether the compound shortest path is detoured by comparing $h_{i(j, k)}$ and $h_{j k}^{*}$. If $h_{i(j, k)}=h_{j k}^{*}$, the compound shortest path corresponds to the direct path between the anchors, and the shortest paths from the normal node to the anchors also correspond to their direct paths. If $h_{i(j, k)}>h_{j k}^{*}$, the compound shortest path is deviated from the direct path between the anchors, and the shortest paths from the normal node to the anchors are detoured. Define the difference between $h_{i(j, k)}$ and $h_{j k}^{*}$ as the path deviation.

The problem here is to find the expected hop count between anchors. The expected hop count between two anchors (an anchor pair) indicates the hop count that is obtainable if nodes are uniformly deployed and any network anisotropy does not exist. Then, the expected hop count between two anchors $j$ and $k$ can be computed with their distance $d_{j k}$ and the mean distance $\mu_{L}$ for a single hop under the uniform node distribution. Since the 


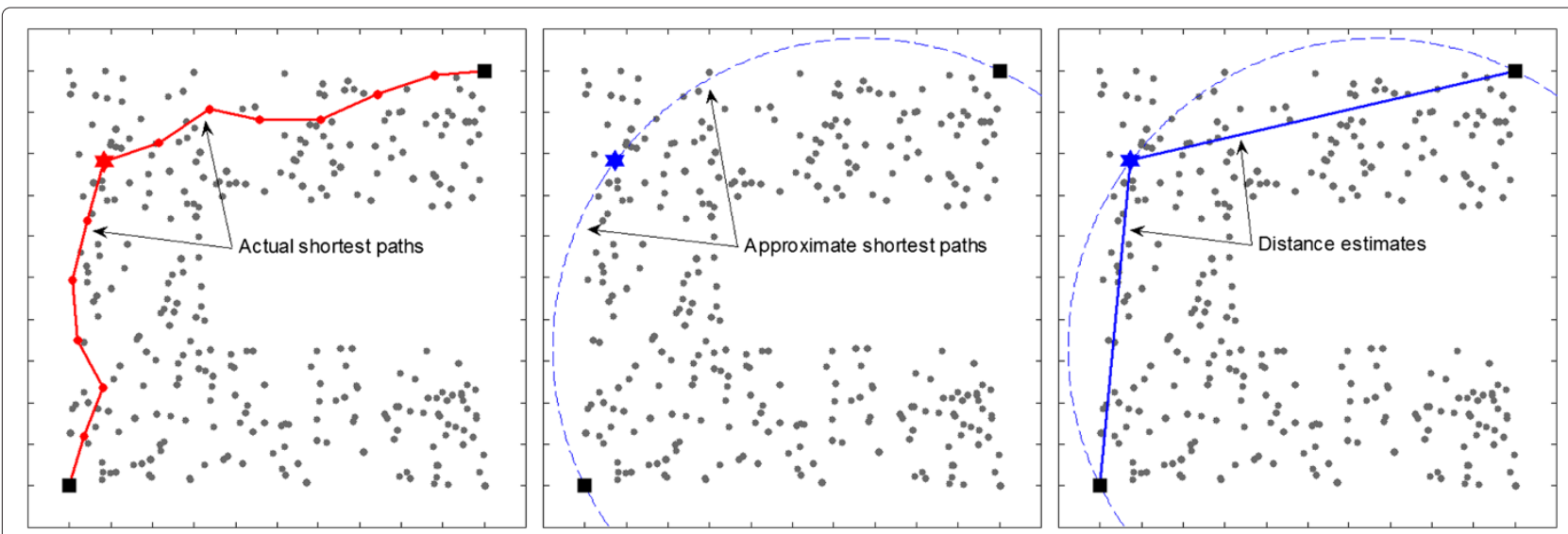

Figure 1 Approximate shortest paths and distance estimations with circular-shaped virtual hole. The proposed algorithm approximates the compound shortest path between two anchors as the boundary of a circular-shaped virtual hole whose size and placement are determined based on the difference between the expected hop count and the hop count of the compound shortest path between the anchors. The distance to each anchor is then estimated by dividing the approximate compound shortest path according to the hop counts to the anchors.

hop count has an integer value, the expected hop count between anchors $j$ and $k$ is derived by

$$
h_{j k}^{*}=\left\lceil\frac{d_{j k}}{\mu_{L}}\right\rceil,
$$

where $\lceil\cdot\rceil$ gives the smallest integer greater than or equal to a number. The mean distance for one hop is computed as

$$
\mu_{L}=\int_{0}^{d_{\max }} \ell p_{L}(\ell) d \ell
$$

where $p_{L}(\ell)$ is the probability density function of the distance between one-hop neighboring nodes, which is given by [18]

$$
p_{L}(\ell)=2 \lambda \pi \ell \exp \left[-\lambda \pi\left(d_{\max }^{2}-\ell^{2}\right)\right]
$$

with node density $\lambda$. However, (2) is defined with the imaginary error function, which cannot be expressed in closed form and needs an approximation for computation. With the Riemann sum [26], (2) can be approximated as

$$
\mu_{L} \approx 2 \lambda \pi\left(\frac{d_{\max }}{\Delta}\right)^{3} \sum_{\delta=0}^{\Delta-1} \delta^{2} \exp \left[-\lambda \pi d_{\max }^{2}\left(1-\frac{\delta^{2}}{\Delta^{2}}\right)\right],
$$

where $\Delta$ is the number of tagged partitions. At each node, the node density can be approximated as $\lambda \approx$ $(n+1) /\left(\pi d_{\max }^{2}\right)$; as a result, the mean distance computed by each normal node can be different. Throughout this paper, we omit the subscript indicating normal nodes in the expected hop count and the mean distance.
3.3 Distance estimation with approximate shortest path Our problem is to build a virtual hole between an anchor pair given the path deviation (i.e., the difference between the hop counts of the anchor pair's direct and compound shortest paths) in order to measure how much the shortest path from a normal node to an anchor is detoured. Recall that a circular-shaped virtual hole is considered in this paper. Consider that normal node $i$ observes the compound shortest path of an anchor pair $j$ and $k$. Let $\mathbf{O}_{i(j, k)}=$ $\left[x_{i(j, k)}, y_{i(j, k)}\right]^{T}$ and $r_{i(j, k)}$ be the center and radius of the virtual hole, respectively, which is placed between the $j-k$ anchor pair by normal node $i$. Since the shortest path is drawn along the boundary of a hole [19], the compound shortest path can be drawn along the arc of a circular sector in our circular-shaped virtual hole model. Denote the length of the arc (i.e., the compound shortest path) and the central angle of the circular vector by $l_{i(j, k)}$ and $\omega_{i(j, k)}$, respectively. The geometric parameters are illustrated in Figure 2.

The shortest path represents the total travel distance of a packet. Hence, the length of the shortest path is approximately as follows:

$$
l_{i(j, k)} \approx \begin{cases}d_{j k} & \text { if } h_{i(j, k)}=h_{j k}^{*} \\ \mu_{L} h_{i(j, k)} & \text { if } h_{i(j, k)}>h_{j k}^{*}\end{cases}
$$

From the geometry, the following relationships are readily obtained:

$$
\begin{aligned}
& l_{i(j, k)}=\omega_{i(j, k)} r_{i(j, k)}, \\
& d_{j k}=2 r_{i(j, k)} \sin \left(\frac{\omega_{i(j, k)}}{2}\right) \quad \text { for } \quad \omega_{i(j, k)} \in(0,2 \pi) .
\end{aligned}
$$



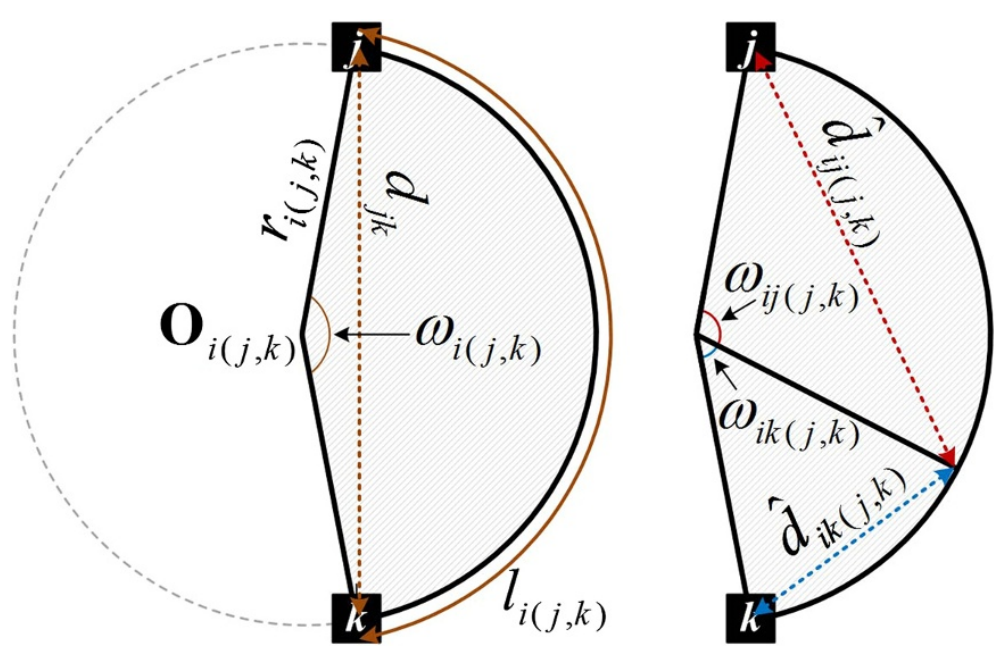

Figure 2 Geometric representation of approximate shortest path with circular-shaped virtual hole.

From the feasible region of the central angle, $r_{i(j, k)} \in$ $\left[d_{j k} / 2, \infty\right)$, and (6) is rearranged in terms of the central angle to yield

$$
\sin \left(\frac{\omega_{i(j, k)}}{2}\right)=\frac{d_{j k} \omega_{i(j, k)}}{2 l_{i(j, k)}}
$$

Since there is no closed-form solution to (7), we transform it into an optimization problem to be solved by an optimization algorithm such as gradient and Newton's methods:

$$
\min f\left(\omega_{i(j, k)}\right)=\left|\sin \left(\frac{\omega_{i(j, k)}}{2}\right)-\frac{d_{j k} \omega_{i(j, k)}}{2 l_{i(j, k)}}\right|^{2}
$$

subject to $\omega_{i(j, k)} \in(0,2 \pi)$.

Because this constrained optimization problem is convex, the optimal central angle, denoted by $\omega_{i(j, k)}^{*}$, for the circular sector can be obtained by updating the central angle with an optimization algorithm until it converges. After the central angle is determined, the radius of the virtual hole can be obtained from (6). The center of the virtual hole is easily derived because the distances from the center to the anchors are identical. There are two solutions to the problem of finding the center of the virtual hole, and the two solutions are symmetric to each other with respect to the straight line connecting the anchor pair. As a result, two possible shortest paths, which are also symmetric to each other, can be drawn. Due to the symmetric property, the distances from any points on the shortest path to the anchors are identical to the distances from the symmetric points to the anchors, irrespective of the placement of the virtual hole.
By constructing the virtual hole based on the path deviation, the compound shortest path is approximated as the arc of the circular sector of the virtual hole. Recall that the compound shortest path of the anchor pair is the path connecting the two shortest paths from the normal node to the anchors. The circular sector with central angle $\omega_{i(j, k)}^{*}$ then can be divided into two subsectors such that the arcs of the two subsectors correspond to the shortest paths to the anchors as seen in Figure 2. The central angles of the two subsectors are calculated by

$$
\begin{aligned}
& \omega_{i j(j, k)}=\frac{h_{i j}}{h_{i(j, k)}} \omega_{i(j, k)}^{*}, \\
& \omega_{i k(j, k)}=\frac{h_{i k}}{h_{i(j, k)}} \omega_{i(j, k)}^{*} .
\end{aligned}
$$

The distances from normal node $i$ to anchors $j$ and $k$ are estimated with the cosine rule which are given by

$$
\begin{aligned}
& \hat{d}_{i j(j, k)}=r_{i(j, k)} \sqrt{2\left(1-\cos \omega_{i j(j, k)}\right)}, \\
& \hat{d}_{i k(j, k)}=r_{i(j, k)} \sqrt{2\left(1-\cos \omega_{i k(j, k)}\right)} .
\end{aligned}
$$

Repeating the above procedure for different anchor combinations, normal node $i$ acquires $A-1$ distance estimates for the distance to an anchor, and the final distance estimate to anchor $j$ is determined as follows:

$$
\hat{d}_{i j}=\sum_{k \neq j} w_{i j(j, k)} \hat{d}_{i j(j, k)}
$$


where $j, k \in \Omega_{A}$ and $w_{i j(j, k)}$ is the weight of distance estimate $\hat{d}_{i j(j, k)}$. Since the approximate shortest paths are not accurate physical representations, the distance estimates may be inaccurate with heavily detoured paths due to the variety of the paths. Another reason for inaccurate distance estimation in the proposed algorithm is the path duplicate problem; the separately measured shortest paths from the normal node to the anchor pair are duplicated with each other. Due to the path duplicate, the shortest paths to the anchors are considered to be more detoured than the actual paths. To minimize the effects of the detoured paths and the path duplicate problem in distance estimation, a two-stage weight allocation is proposed. In the first stage, the weight $\dot{w}_{i j(j, k)}$ is computed as

$$
\dot{w}_{i j(j, k)} \propto \frac{1}{\left|h_{j k}-h_{i(j, k)}\right|^{2}},
$$

where the difference between $h_{j k}$ and $h_{i(j, k)}$ represents the possibility of the path duplicate. It is normalized such that $\sum_{k \neq j} \dot{w}_{i j(j, k)}=1$. The second-stage weight is calculated as

$$
\ddot{w}_{i j(j, k)} \propto \frac{\dot{w}_{i j(j, k)}}{\left|h_{j k}^{*}-h_{i(j, k)}\right|^{2}},
$$

where the difference between the expected and measured hop counts represents the extent of the detour of the path. With the weight normalization as in the first stage,

$$
w_{i j(j, k)}=\frac{\ddot{w}_{i j(j, k)}}{\sum_{k \neq j} \ddot{w}_{i j(j, k)}} .
$$

Through the two-stage weight computation, the distance estimate with the shortest path considered to be the direct path has the largest weight.

\subsection{Localization based on least squares estimation}

We briefly explain a least squares localization algorithm in this subsection. From the distance estimates to the anchors, the following linear measurements are obtained:

$$
-2 x_{i}\left(x_{j}-x_{k}\right)-2 y_{i}\left(y_{j}-y_{k}\right)=\hat{d}_{i j}^{2}-\hat{d}_{i k}^{2}-\left\|\mathbf{p}_{j}\right\|^{2}+\left\|\mathbf{p}_{k}\right\|^{2},
$$

for $j=1,2, \ldots, A-1, k=j+1, j+2, \ldots, A$, and $j, k \in \Omega_{A}$. The location of normal node $i$ is estimated with the least squares estimation,

$$
\hat{\mathbf{p}}_{i}=\left(\mathbf{H}^{T} \mathbf{H}\right)^{-1} \mathbf{H}^{T} \mathbf{z}_{i}=\left[\hat{x}_{i}, \hat{y}_{i}\right]^{T}
$$

where measurement matrix $\mathbf{H}$ is

$$
\mathbf{H}=-2 \times\left[\left(\mathbf{p}_{1}-\mathbf{p}_{2}\right) \cdots\left(\mathbf{p}_{j}-\mathbf{p}_{k}\right) \cdots\left(\mathbf{p}_{A-1}-\mathbf{p}_{A}\right)\right]^{T}
$$

and measurement vector $\mathbf{z}_{i}$ is

$$
\mathbf{z}_{i}=\left[\begin{array}{c}
\hat{d}_{i 1}^{2}-\hat{d}_{i 2}^{2}-\left\|\mathbf{p}_{1}\right\|^{2}+\left\|\mathbf{p}_{2}\right\|^{2} \\
\vdots \\
\hat{d}_{i j}^{2}-\hat{d}_{i k}^{2}-\left\|\mathbf{p}_{j}\right\|^{2}+\left\|\mathbf{p}_{k}\right\|^{2} \\
\vdots \\
\hat{d}_{i A-1}^{2}-\hat{d}_{i A}^{2}-\left\|\mathbf{p}_{A-1}\right\|^{2}+\left\|\mathbf{p}_{A}\right\|^{2}
\end{array}\right] .
$$

The proposed algorithm running on each normal node $i$ is summarized in Algorithm 1 .

\section{Algorithm 1 Multihop range-free localization with approximate shortest path \\ Input:}

Anchor knowledge set received from anchors $j$ and $k$ : the location information of the anchors and minimum hop count to each anchor, stored as $\left\{\mathbf{p}_{j}, \mathbf{p}_{k}, h_{i j}, h_{i k}\right\}$;

Output: Location estimate $\hat{\mathbf{p}}_{i}$ of node $i$.

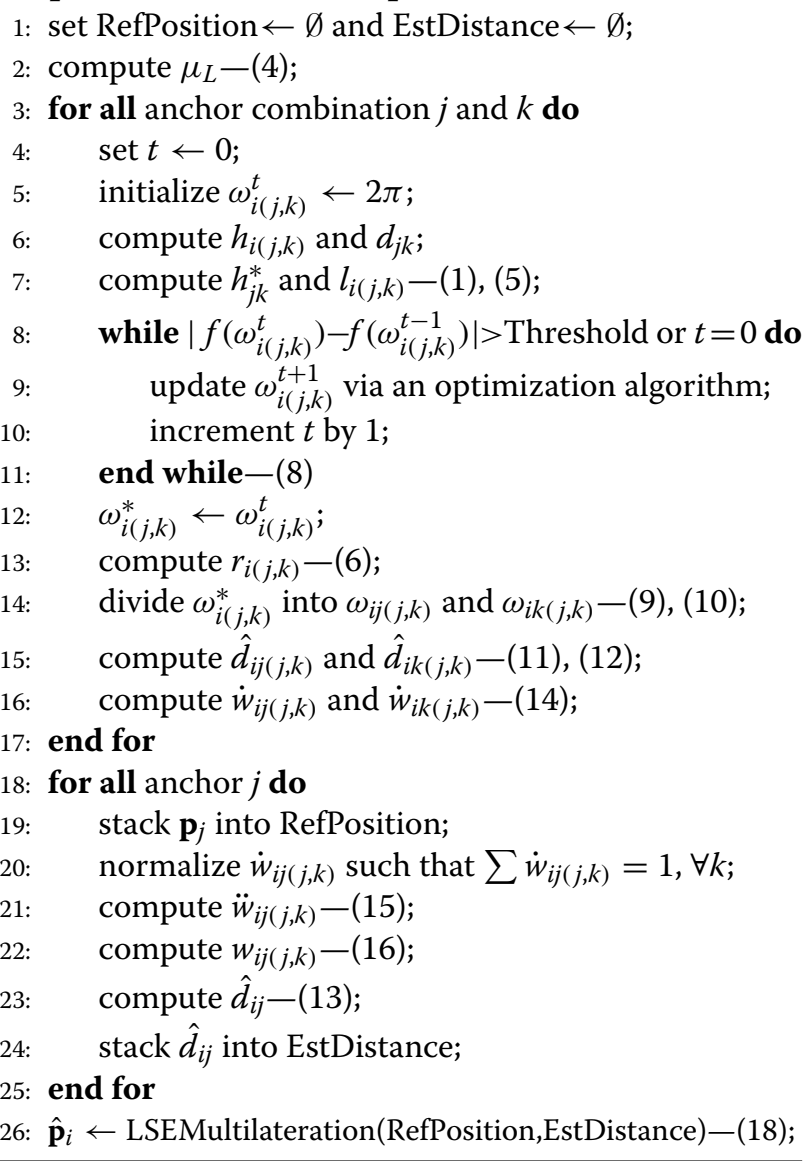




\section{Performance evaluation and analysis}

In this section, we show the effectiveness of the proposed algorithm via MATLAB simulations. The estimation accuracy of the proposed algorithm is compared to those of the DV-Hop [11], LAEP [18], and supervised anchor [21] algorithms under varying network configurations. Other algorithms that run as the DV-Hop algorithm without specific network configurations are not included in the performance comparison. To evaluate and compare the performances of the range-free localization algorithms, the distance and location estimation errors are computed. The normalized error of distance estimate from normal node $i$ to anchor $j$ is calculated by $\varepsilon_{i j}=\mid \hat{d}_{i j}-$ $d_{i j} \mid / d_{\max }$ for $i \in \Omega_{N}, j \in \Omega_{A}$. Note that $\hat{d}_{i j} \neq 0$. The normalized localization error of normal node $i$ with distance estimates to at least three anchors is $\gamma_{i}=\left\|\hat{\mathbf{p}}_{i}-\mathbf{p}_{i}\right\| / d_{\max }$.

We randomly distribute 300 normal nodes over $10 d_{\max } \times 10 d_{\max }$ irregular regions and vary anchor numbers from 4 to 14 . The irregular regions used in the simulations are the $\mathrm{C}$-shaped, E-shaped, and S-shaped regions as shown in Figure 3. In addition to the large hole, small holes may exist as well due to the nonuniform node distribution and radio irregularity. As mentioned in Section 2, the QUDG model is adopted for irregular radio propagation.

\subsection{Path deviation and communication overhead}

Figure 4 shows how the path deviation, which is computed as the difference between the expected hop count and measured hop count of an anchor-to-normal pair, changes with the increase of the DOI ratio and the hop count. The path deviation represents the extent of the detour of the shortest path between a normal node and an anchor.

The path deviation increases as the hop count increases and radio irregularity becomes more severe. This indicates that the shortest path between a node pair is more likely detoured at high DOI ratio and large hop count. In particular, for DOI $=2$, the nodes that are 30 hops away from anchors can, in fact, reach the anchors within approximately 8 hops in the absence of the network anisotropy. The path deviation for the nodes at 30 hops is approximately 19 hops even without radio irregularity. Therefore, the need of the detoured path detection is emphasized for nodes that large hops away from anchors even though the effect of radio irregularity is negligible.

The communication overhead of the proposed algorithm is bounded by $2 O(A N)$ as well as the DV-Hop and supervised anchor algorithms. These algorithms perform flooding twice where each flooding has a communication overhead of $O(A N)$; each node in the network forwards a flooding packet, originated by an anchor, at least once. Each flooding is carried out

1. to broadcast the location information of anchors and to learn the hop counts between nodes and anchors; and

2. to broadcast the anchors' average hop progresses in the DV-Hop and supervised anchor algorithms or to inform the hop counts between the anchors in the proposed algorithm.

The communication overhead of the LAEP algorithm is $O(A N)$ because flooding is implemented only once in the LAEP algorithm. Although the proposed algorithm has a twofold communication overhead of the LAEP algorithm, its overhead is insignificant compared to the other algorithms.

\subsection{Distance estimation error}

Figures 5, 6, 7 show how distance estimation errors of the DV-Hop, LAEP, supervised anchor, and proposed algorithms change with the increase of the hop count in different irregular regions. In the simulations,
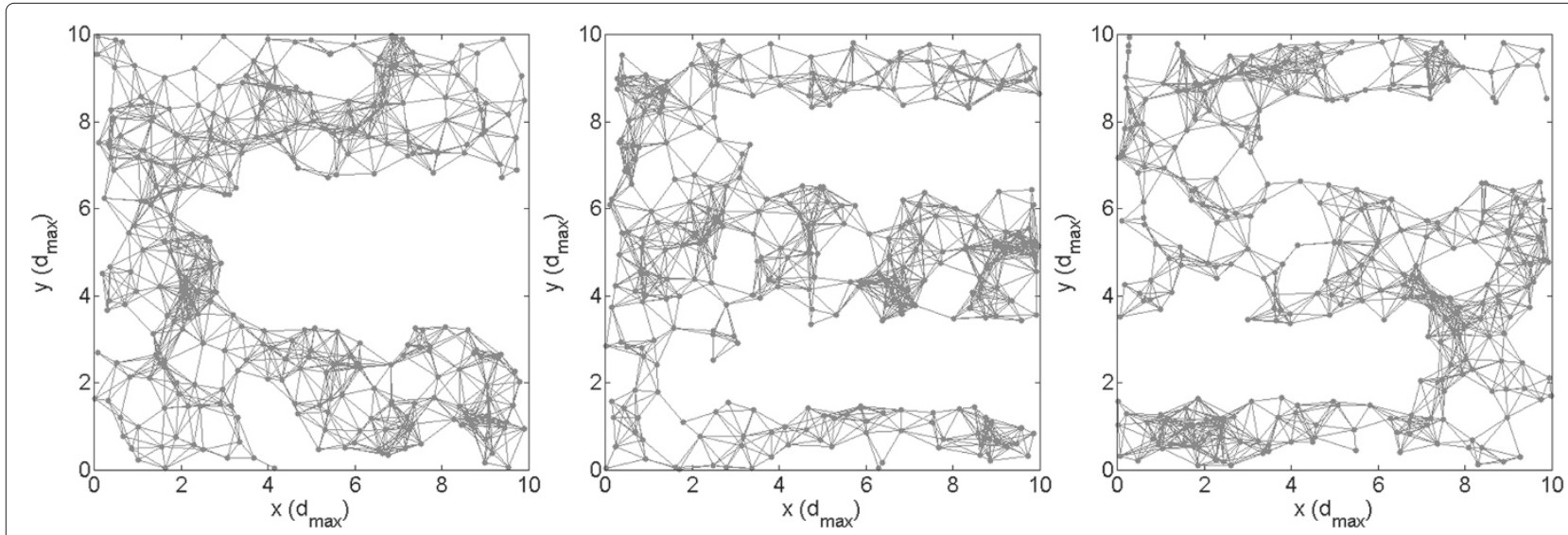

Figure 3 Exemplary topologies under irregular regions. In irregular regions, one or two large holes are placed and nodes are deployed away from the holes. According to the placements of the large holes, C-shaped (left), E-shaped (middle), and S-shaped (right) networks are formed. 


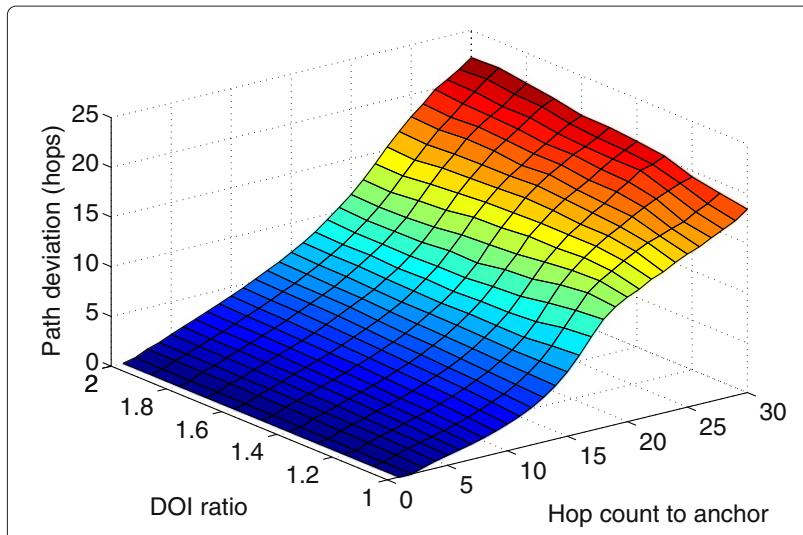

Figure 4 Path deviation with respect to $\mathrm{DOI}$ ratios and hop counts.

DOI $=1.5$ and 14 anchors are randomly deployed in the C-shaped, E-shaped, and S-shaped regions. As seen in these figures, the distance estimation accuracy of the range-free algorithms decreases as the hop count increases. The performance of the DV-Hop, LAEP, and supervised anchor algorithms starts to deteriorate dramatically at around 15 hops. By using the DV-Hop, LAEP, and supervised anchor algorithms, the distance estimation errors at 30 hops reach approximately $5.4 d_{\max }$, $10.5 d_{\max }$, and $6.6 d_{\max }$ in the C-shaped region; $3.8 d_{\max }$, $4.5 d_{\max }$, and $6.3 d_{\max }$ in the E-shaped region; and $3.6 d_{\max }$, $8.8 d_{\max }$, and $3.7 d_{\max }$ in the $S$-shaped region. In contrast to the three localization algorithms, the proposed algorithm achieves the distance estimation errors less than $2 d_{\max }$ regardless of the hop counts and the regions. Through these simulations, it is demonstrated that the proposed algorithm significantly improves the accuracy of distance estimates in anisotropic networks.

To evaluate the performance of the range-free algorithms with a small number of anchors, 4 anchors are

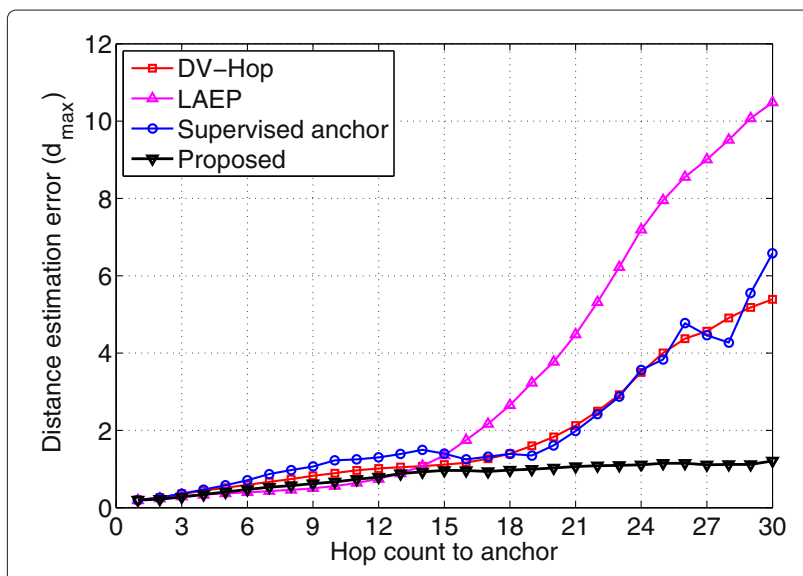

Figure 5 Distance estimation errors of range-free localization algorithms in C-shaped regions with 14 anchors.

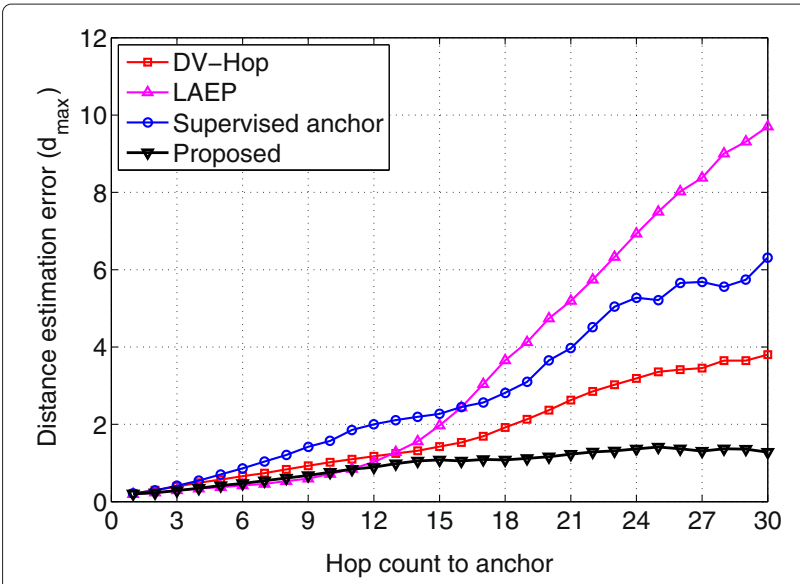

Figure 6 Distance estimation errors of range-free localization algorithms in E-shaped regions with 14 anchors.

randomly distributed in the S-shaped regions and DOI $=$ 1.5. The result is presented in Figure 8. Compared with the result in Figure 7, the distance estimation errors of the range-free algorithms, except for the LAEP algorithm, increase. This is because that the LAEP algorithm uses only local node density, which is stationary with change in anchor numbers, in distance estimations. Even though the performance of the proposed algorithm is degraded with the reduced number of anchors, it still provides distance estimates with significantly less errors compared to the other algorithms. Furthermore, the proposed algorithm achieves better performance with only 4 anchors than the other algorithms using 12 anchors.

Although the LAEP and supervised anchor algorithms were proposed for anisotropic sensor networks, they do not show any significant performance improvement over the DV-Hop algorithm in the simulations. As the LAEP algorithm copes with nonuniform node distribution, it

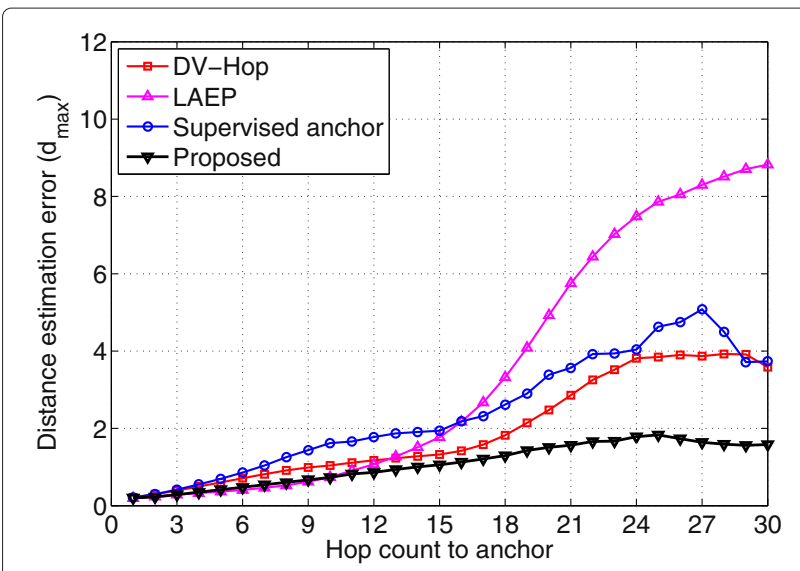

Figure 7 Distance estimation errors of range-free localization algorithms in S-shaped regions with 14 anchors. 


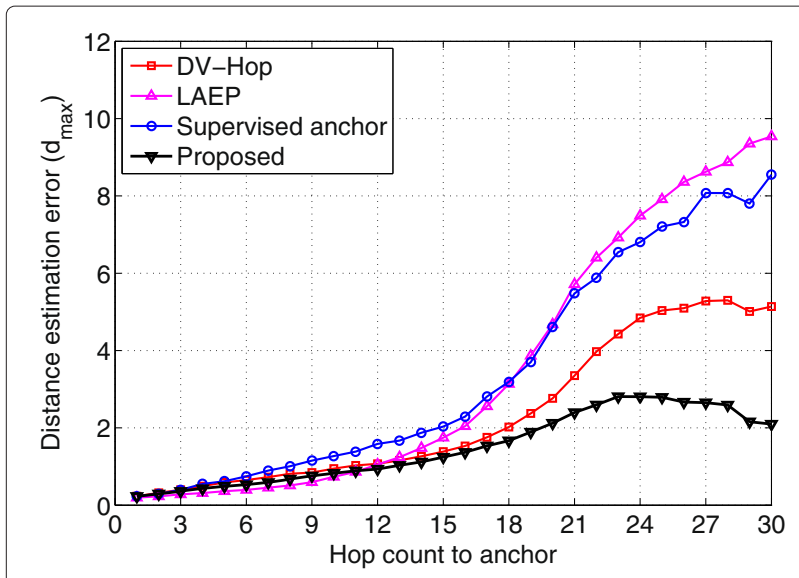

Figure 8 Distance estimation errors of range-free localization algorithms in S-shaped regions with 4 anchors.

is able to estimate the distance between one-hop neighboring nodes precisely. However, the distance between multihop-away nodes is determined as the sum of the distance estimates between one-hop neighboring nodes without any consideration of other network anisotropy. For this reason, the LAEP algorithm encounters avalanche errors in distance estimations for nodes having large hops to anchors. The supervised anchor algorithm allows the nodes that are one-hop away from the anchors to use the best anchor subsets determined by the one-hop neighboring anchors. Since the other nodes use the anchor subset that are obtained based on the location estimates of onehop neighboring normal nodes, improper anchor subsets can be derived. Numerous anchors are required to prevent this problem, but since the number of anchors is far less than the number of normal nodes in the simulations, considerably large errors are observed as well as the LAEP algorithm.

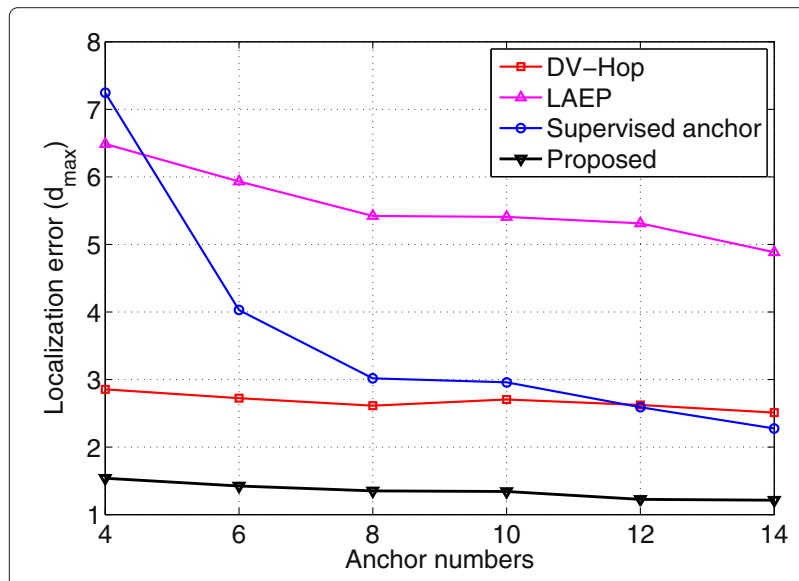

Figure 9 Localization errors of range-free localization algorithms with respect to anchor numbers in C-shaped region.

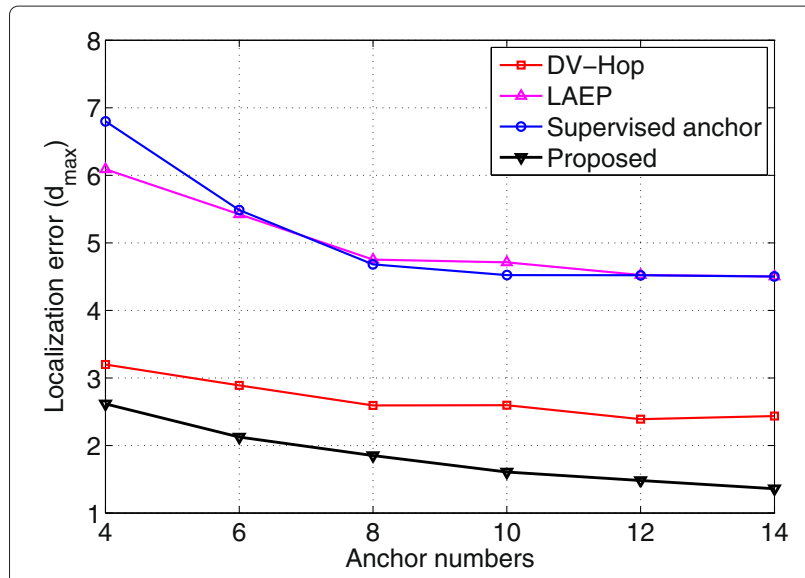

Figure 10 Localization errors of range-free localization algorithms with respect to anchor numbers in E-shaped region.

\subsection{Localization error}

Figures 9, 10, 11 show the localization errors of the rangefree algorithms by varying the number of anchors in the C-shaped, E-shaped, and S-shaped regions, respectively. The DOI ratio is set to 1.5 in the simulations. The localization accuracy of the range-free algorithms is enhanced as the number of anchors increases. Especially, the localization errors of the supervised anchor algorithm are rapidly reduced by increasing the number of anchors. This is because nodes are able to effectively select a set of anchors whose paths are rarely detoured when a sufficient number of anchors are deployed in the supervised anchor algorithm. Since the proposed algorithm can detect the detoured path with a small number of anchors, it has higher localization accuracy than the other localization algorithms in any conditions. The proposed algorithm achieves similar or even higher localization accuracy with 4 anchors than the other algorithms with 14 anchors in

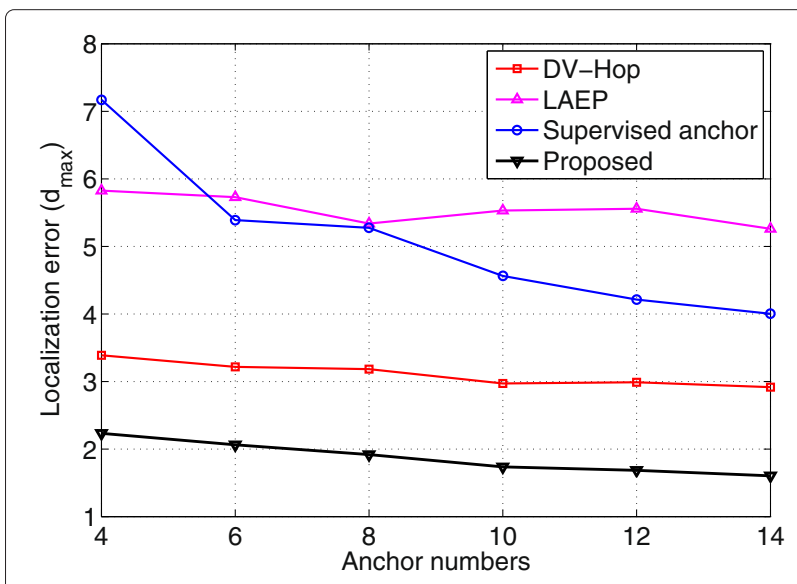

Figure 11 Localization errors of range-free localization algorithms with respect to anchor numbers in S-shaped region. 


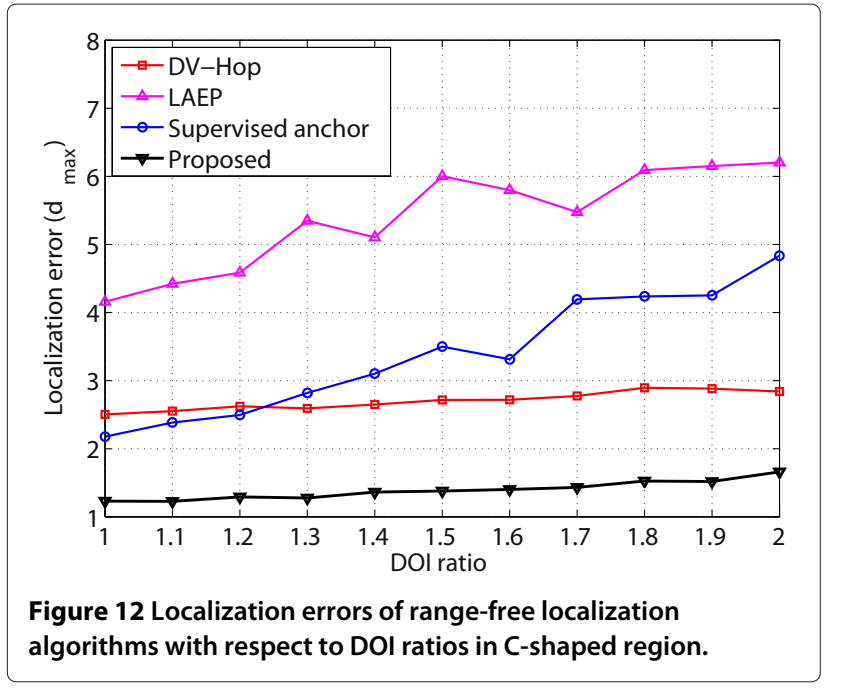

the C-shaped, E-shaped, and S-shaped regions. The simulation results showed the network anisotropy can be handled with only few anchors by using the proposed algorithm.

Performance of the range-free localization algorithms under different DOI ratios is evaluated, and the results are shown in Figures 12, 13, 14. In the simulations, the DOI ratios vary from 1 to 2 . Recall that the link connection is perfectly established between nodes within the maximum transmission radius from each other when DOI = 1 , and the distance that guarantees a perfect link between nodes is reduced to half of the maximum transmission radius for $\mathrm{DOI}=2$. Therefore, the shortest path between nodes is more likely detoured as the DOI ratio increases (see Figure 4). The increase of detoured paths at high DOI ratios leads to performance degradation of the range-free

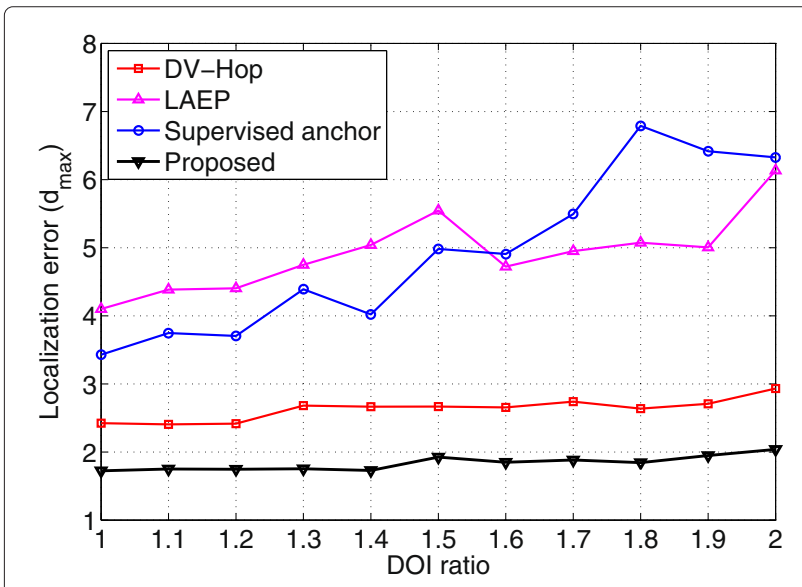

Figure 13 Localization errors of range-free localization algorithms with respect to DOI ratios in E-shaped region.

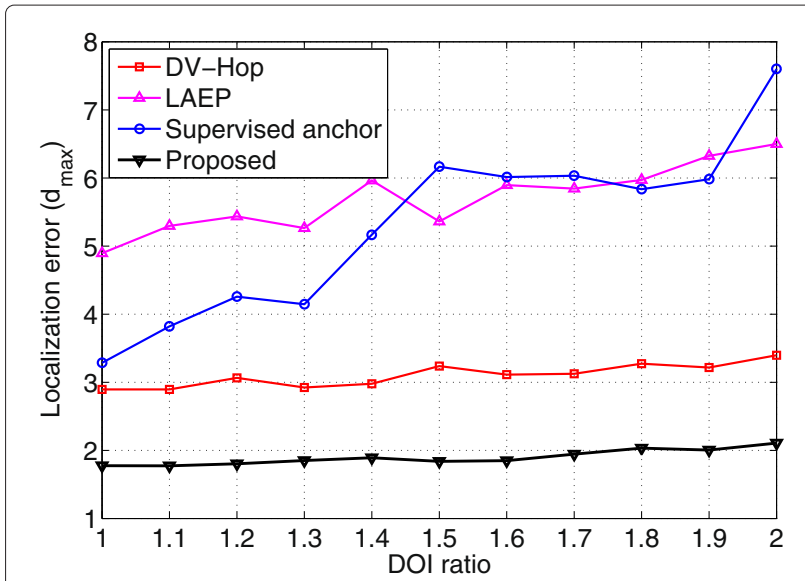

Figure 14 Localization errors of range-free localization algorithms with respect to $\mathrm{DOI}$ ratios in S-shaped region.

algorithms as shown in the figures. It has to be emphasized that the performance degradation of the proposed algorithm from radio irregularity is marginal while the performance of the other algorithms is degraded dramatically. Moreover, regardless of the DOI ratios, the localization errors of the proposed algorithm are less than or nearly equal to $2 d_{\text {max }}$; whereas, the other algorithms provide location estimates with errors over $2 d_{\max }$ even in the case of DOI $=1$.

Figures 15, 16, 17, 18 show the localization errors of the range-free algorithms at different node locations under $\mathrm{DOI}=2$. For the ease of visualization, the simulations were conducted only in the $\mathrm{C}$-shaped region. The localization errors of the proposed algorithm are mostly less than those of the DV-Hop, LAEP, and supervised anchor algorithms at any locations. Especially, the localization accuracy at the hole boundaries and at the beginning and end of ' $\mathrm{C}$ ' is significantly improved with the proposed algorithm. Nodes located at those areas tend to have heavily detoured shortest paths to distant anchors.

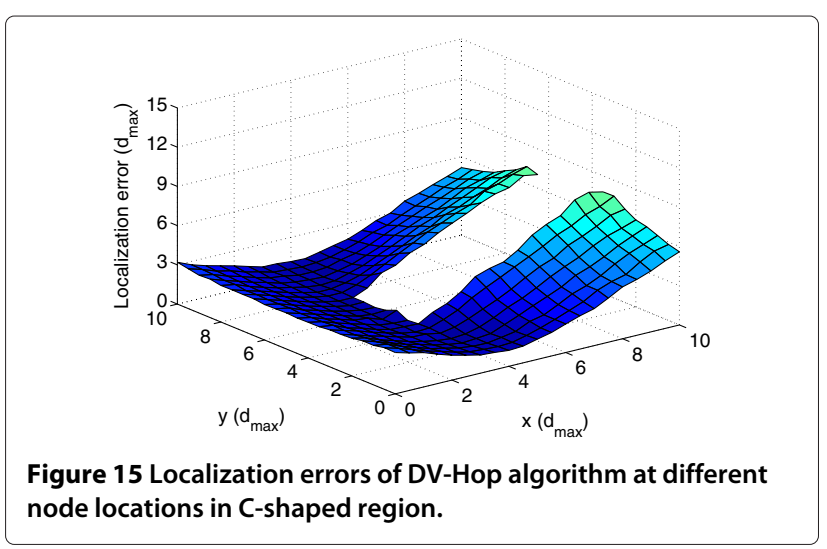




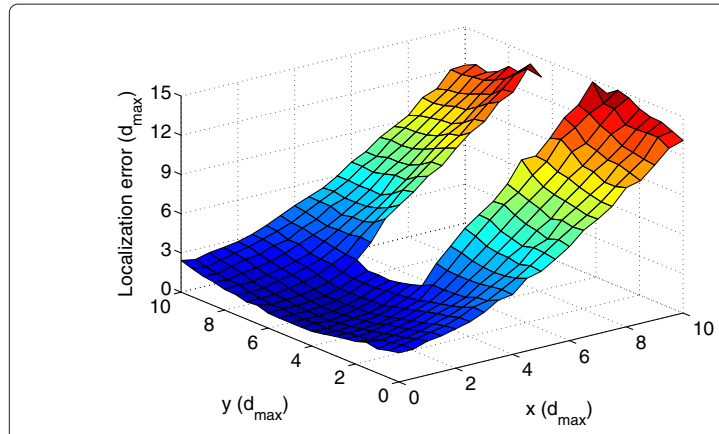

Figure 16 Localization errors of LAEP algorithm at different node locations in C-shaped region.

At the corners of the C-shaped region, the localization errors of the proposed algorithm are higher than those at the other locations. It is possible that the performance at the corners is relatively poor because the shortest paths to most of the anchors are duplicated with each other. Nevertheless, the proposed algorithm achieves better or similar performance compared to the other algorithms.

\section{Conclusions}

A multihop range-free localization algorithm with approximate shortest paths was proposed for anisotropic networks. In the proposed algorithm, each normal node approximates the shortest paths to two arbitrary anchors with a virtual hole placed between the anchors and estimates the distances to the anchors by considering how much their approximate shortest paths are detoured. By using approximate shortest paths, the proposed algorithm can effectively handle with the anisotropic network localization problem with fewer anchors compared to existing range-free algorithms. Through extensive computer simulations, we demonstrated that the proposed algorithm enhances the distance estimation accuracy and achieves

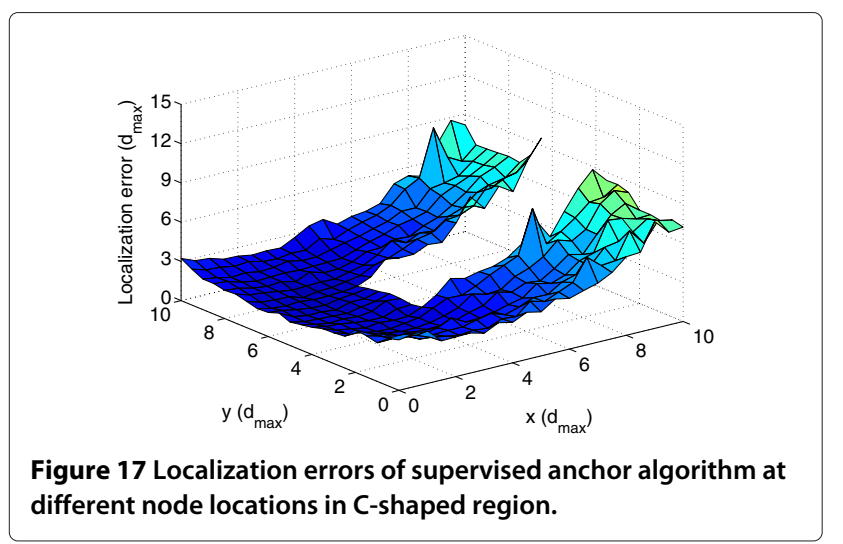

improved localization performance than other range-free algorithms with a small number of anchors.

\section{Competing interests}

The authors declare that they have no competing interests.

\section{Acknowledgements}

This work has been supported by the National GNSS Research Center Program of Defense Acquisition Program Administration and Agency for Defense Development and by the National Research Foundation of Korea (NRF-2013R1A1A2062728) grant funded by the Korea government. A preliminary version of this article was appeared in Korea Information and Communication Society (KICS) Journal 2013. This version includes a weighted sum distance estimation for general wireless sensor networks and an extended analysis of the simulation results.

\section{Author details}

${ }^{1}$ Department of Electronics and Computer Engineering, Hanyang University, Seoul 133-791, Republic of Korea. ${ }^{2}$ School of Electronics Engineering, Chungbuk National University, Cheongju 361-763, Republic of Korea. ${ }^{3}$ Agency for Defense Development, Daejeon 305-152, Republic of Korea.

Received: 12 November 2013 Accepted: 13 April 2014

Published: 16 May 2014

\section{References}

1. B Rao, L Minakakis, Evolution of mobile location-based services. Commun. ACM. 46(12), 61-65 (2003)

2. S Lee, C Lee, ed. by X Wang, Broadcasting in mobile ad hoc networks, in Mobile Ad-Hoc Networks: Protocol Design (InTech, India, 2011), pp. 579-594

3. L Barriére, P Fraigniaud, L Narayanan, Robust position-based routing in wireless ad hoc networks with unstable transmission ranges. Paper presented at the ACM Dial-M, (Rome, Italy, 21 July 2001), pp. 19-27

4. AS Paul, EA Wan, RSSI-based indoor localization and tracking using sigma-point Kalman smoother. IEEE J. Sel. Topics Signal Process. 3(5), 860-873 (2009)

5. SD Chitte, S Dasgupta, Z Ding, Distance estimation from received signal strength under log-normal shadowing: bias and variance. IEEE Signal Process. Lett. 16(3), 216-218 (2009)

6. X Li, K Pahlavan, Super-resolution TOA estimation with diversity for indoor geolocation. IEEE Trans. Wireless Commun. 3(1), 224-234 (2004)

7. L Yang, KC Ho, Alleviating sensor position error in source localization using calibration emitters at inaccurate locations. IEEE Trans. Signal Process. 58(1), 61-83 (2010)

8. Y Shen, MZ Win, On the accuracy of localization systems using wideband antenna arrays. IEEE Trans. Commun. 58(1), 270-280 (2010)

9. N Bulusu, J Heidemann, D Estrin, GPS-less low-cost outdoor localization for very small devices. IEEE Pers. Commun. 7(5), 28-34 (2000)

10. THe, C Huang, BM Blum, BM Stankovic, T Abdelzaher, Range-free localization schemes for large scale sensor networks. Paper presented at the ACM MobiCom (San Diego, CA, USA, 14-19 Sept 2003), pp. 81-95 
11. D Niculescu, B Nath, Ad hoc positioning system (APS). Paper presented at the IEEE GLOBECOM (San Antonio, TX, USA, 25-29 Nov 2001), pp. 2926-2931

12. Y Shang, W Ruml, Y Zhang, MPJ Fromherz, Localization from mere connectivity. Paper presented at the ACM MobiHoc, (Annapolis, MD, USA, 1-3 June 2003), pp. 201-212

13. Y Shang, W Ruml, Improved MDS-based localization. Paper presented at the IEEE INFOCOM (Hong Kong, 7-11 Mar 2004), pp. 2640-2651

14. Q Xiao, B Xiao, J Cao, J Wang, Multihop range-free localization in anisotropic wireless sensor networks: a pattern-driven scheme. IEEE Trans. Mobile Comput. 9(11), 1592-1607 (2010)

15. AA Kannan, B Fidan, G Mao, Use of flip ambiguity probabilities in robust sensor network localization. Wirel. Netw. 17(5), 1157-1171 (2011)

16. S Lee, H Woo, C Lee, Wireless sensor network localization with connectivity-based refinement using mass spring and Kalman filtering. EURASIP J. Wirel. Comm. 2012(1), 152 (2012)

17. NB Priyantha, H Balakrishnan, E Demaine, S Teller, Anchor-free distributed localization in sensor networks (2003). LCS Technical Report 892. MIT Laboratory for Computer Science. http://nms.lcs.mit.edu/cricket

18. Y Wang, X Wang, D Wang, DP Agrawal, Range-free localization using expected hop progress in wireless sensor networks. IEEE Trans. Parallel Distrib. Syst. 20(10), 1540-1552 (2009)

19. $M$ Li, Y Liu, Rendered path: range-free localization in anisotropic sensor networks with holes. IEEE/ACM Trans. Netw. 18(1), 320-332 (2010)

20. B Xiao, L Chen, Q Xiao, M Li, Reliable anchor-based sensor localization in irregular areas. IEEE Trans. Mobile Comput. 9(1), 60-72 (2009)

21. X Liu, S Zhang, J Wang, J Cao, B Xiao, Anchor supervised distance estimation in anisotropic wireless sensor networks. Paper presented at the IEEE WCNC (Quintana Roo, Mexico, 28-31 Mar 2011), pp. 938-943

22. Y Wang, J Gao, Boundary recognition in sensor networks by topological methods. Paper presented at the ACM MobiCom (Los Angeles, CA, USA, 24-29 Sept 2006), pp. 122-133

23. V-s Fang, SY Chang, Determination of wireless networks parameters through parallel hierarchical support vector machines. IEEE Trans. Parallel Distrib. Syst. 23(3), 505-512 (2012)

24. J Lee, B Choi, E Kim, Novel range-free localization based on multidimensional support vector regression trained in the primal space. IEEE Neural Netw. Learn. Syst. 24(7), 1099-1113 (2013)

25. D Gao, P Chen, C Foh, Y Niu, Hop-distance relationship analysis with quasi-UDG model for node localization in wireless sensor networks. EURASIP J. Wirel. Comm. 2011(1), 99 (2011)

26. JA Fridy, Introductory Analysis: The Theory of Calculus. (Academic, San Diego, 2000)

doi:10.1186/1687-1499-2014-80

Cite this article as: Lee et al: Multihop range-free localization with approximate shortest path in anisotropic wireless sensor networks. EURASIP Journal on Wireless Communications and Networking 2014 2014:80.

\section{Submit your manuscript to a SpringerOpen ${ }^{\mathcal{O}}$ journal and benefit from:}

- Convenient online submission

- Rigorous peer review

- Immediate publication on acceptance

- Open access: articles freely available online

- High visibility within the field

- Retaining the copyright to your article

Submit your next manuscript at $\boldsymbol{\wedge}$ springeropen.com 